\title{
Hard diffraction in PythiA 8
}

\author{
Christine Overgaard Rasmussen ${ }^{1, a}$ \\ ${ }^{1}$ Theoretical Particle Physics, \\ Department of Astronomy and Theoretical Physics, \\ Lund University, \\ Sölvegatan 14A, \\ SE-22362 Lund, \\ Sweden.
}

\begin{abstract}
We present an overview of the options for diffraction implemented in the general-purpose event generator Pутніа 8 [1]. We review the existing model for soft diffraction and present a new model for hard diffraction. Both models use the Pomeron approach pioneered by Ingelman and Schlein, factorising the diffractive cross section into a Pomeron flux and a Pomeron PDF, with several choices for both implemented in PyтніA 8. The model of hard diffraction is implemented as a part of the multiparton interactions (MPI) framework, thus introducing a dynamical gap survival probability that explicitly breaks factorisation.
\end{abstract}

\section{Introduction}

Despite the great success of QCD many soft phenomena still remain as mysteries because of their nonperturbative origin. Amongst these lies soft hadron collisions, in which one or both of the hadrons survive the collision. In this regime we cannot predict the collisions or their rate with our usual perturbative framework of Feynman diagrams and factorisation theorems, as these require a breakup of the hadrons into their constituents, which then collide. We must therefore base our work on phenomenological models, many of which has sprung out of theories invented before QCD, such as S-matrix theory and the related Regge theory.

In Regge theory, poles in the plane of complex spin, $\alpha$, can be seen as hadronic resonances. These appear to lie on linear trajectories, $\alpha(t)=\alpha(0)+\alpha^{\prime} t$, of which the most important for high-energy applications is the Pomeron $(\mathbb{P})$ trajectory, with its $\alpha(0)>1$ explaining the rise of the total cross section. The Pomeron must be a colour singlet carrying the quantum numbers of the vacuum, as it should be able to describe elastic scattering of the hadrons. From a more modern viewpoint it would mainly consist of gluons, but a quark content could also be envisioned - as long as this content come in quark-antiquark pairs, in order to preserve the singlet nature of the $\mathbb{P}$. Having defined the state and its possible partonic content, one can perform a topological expansion similar to that of perturbative QCD, where the simplest possible exchange is a single- $\mathbb{P}$ one, describing elastic scattering. More complex processes can be constructed through the triple- $\mathbb{P}$ vertex, resulting in the different

\footnotetext{
a e-mail: christine.rasmussen@thep.lu.se
} 
diffractive topologies. We generally work with three different diffractive processes, all involving the triple- $\mathbb{P}$ vertex; the single-diffractive (SD) processes (one surviving hadron and one triple- $\mathbb{P}$ vertex), double-diffractive (DD) processes (no surviving hadrons, two triple- $\mathbb{P}$ vertices) and central-diffractive (CD) processes (two surviving hadrons and two triple- $\mathbb{P}$ vertices). In this paper we focus on the SD processes, being the simplest possible configuration and the one with the largest cross section.

Ingelman and Schlein proposed a model [2] in which the $\mathbb{P}$ can be viewed as a hadronic state with partonic content. This opened up the possibility for $\mathbb{P}$ parton distribution functions (PDFs) to be combined with the probability of extracting a $\mathbb{P}$ from the colliding hadron, denoted the $\mathbb{P}$ flux. Hence the diffractive system could be envisioned as a hadron-hadron collision at reduced energy, which opened up the possibility for modelling the diffractive processes with existing hadron-hadron event generators.

A model for diffractive systems should be able to describe all aspects of these collisions, such as differential cross sections, one-particle distributions and global event characteristics. The framework used in PyтніA 8 describes all of these aspects in detail. As rapidity gaps are crucial for diffraction, this framework does not permit MPIs in the pp system, as these would fill up the would-have-been rapidity gap. Thus we introduce the concept of rapidity gap survival probability (RGSP) (cite Bjorken), a concept unique to hadron-hadron collisions, given credibility by the lower observed rate for diffraction at the Tevatron as expected from HERA flux and PDF determinations.

\section{Soft diffraction in PythiA 8}

The soft diffraction framework available in PYTHIA 8 was originally developed for its predeccesor, Pyтнia 6 [3], but rewritten and expanded for the new version [4]. The total hadronic cross section is calculated using the Donnachie-Landshoff [5] parametrisation, including both a Pomeron and Reggeon term. The elastic and diffractive cross sections are calculated using the Schuler-Sjöstrand model [6], with the nondiffractive cross section inferred from the above.

The Schuler-Sjöstrand model is based on Regge theory, giving an exponential $t$-dependence and an approximate $d M^{2} / M^{2}$ mass dependence, with fudge factors introduced in order to dampen the cross sections close to the kinematical limits, as well as dampen the DD cross section in regions where the two diffractive systems overlap. Other $\mathbb{P}$-flux models have also been implemented, all of which have been expanded to include both DD and CD where needed [7]. The subsequent evolution and hadronisation of the $\mathbb{P}$ p system is separated from the $\mathbb{P}$-parametrisation, hence being the same for almost all models.

\subsection{Low-mass soft diffraction}

In the low-mass regime, $M \leq 10 \mathrm{GeV}$, the energies are not sufficiently high to apply a perturbative framework to the $\mathbb{P}$ p subsystem. The framework is setup as an interaction, where a $\mathbb{P}$ has "kicked out" a quark or a gluon from the incoming hadron. In the former case a colour string is stretched between the "free" quark and the diquark remnant inside the proton. In the latter case a colour string is stretched between one valence quark inside the hadron through the "free" gluon and back to the diquark in the remnant, resulting in a hairpin-like structure. The probability for the $\mathbb{P}$ to interact in either of the two ways is mass-dependent and tunable, $\mathcal{P}(q) / \mathcal{P}(g)=N / M^{p}$, set up such that the gluons dominate at higher masses. Since this is a nonperturbative framework, no additional evolution of the colour strings is applied. Instead the system is hadronised directly using the Lund string fragmentation model [8], thus giving rise only to low- $p_{\perp}$ activity in the diffractive system. 


\subsection{High-mass soft diffraction}

In the high-mass regime, $M>10 \mathrm{GeV}$, energies are sufficiently high to use a perturbative framework. The high-mass regime is smoothly connected to the low-mass one, with the fraction of perturbative events gradually increasing from the $10 \mathrm{GeV}$ starting point and dominating at diffractive masses above $20 \mathrm{GeV}$. In this regime the $\mathbb{P}$ is viewed as having a partonic content. Thus once $M$ and $t$ have been selected according to the model of the $\mathbb{P}$ flux, the $\mathbb{P}$ p subsystem is set up and a semi-hard perturbative $2 \rightarrow 2$ QCD process is selected by the MPI framework as the hardest collision. The subsystem is then further evolved using the interleaved parton evolution of PутніA 8, including both MPI as well as initial and final state showers (ISR, FSR), using the $\mathbb{P}$ PDFs on the diffractive side.

The MPI acitivity in the $\mathbb{P p}$ system has been tuned to give approximately the same amount of activity as nondiffractive events of the same mass by introducing an effective total $\mathbb{P}$ cross section of $10 \mathrm{mb}$. This is a tunable parameter, and can be made to depend on the mass of the diffractive system, just as the total pp cross section does. The evolution results in a larger number of colour strings in the event, all hadronised with the Lund string fragmentation model, giving rise to low- to high- $p_{\perp}$ activity in the event.

Although largely successful, the model for soft diffraction in PYтнIA 8 has minor issues. Not all aspects of data are described by the default model and settings, including both differential cross sections and particle spectra. A full retune could improve some of these discrepancies, but a more flexible shape for the $\mathbb{P}$ flux may be needed in order to describe data better. Such studies are planned for the near future.

\section{Hard diffraction in Pythia 8}

The model described in the previous section allows for $2 \rightarrow 2$ QCD processes at all $p_{\perp}$ scales, but is primarily intended for lower values of $p_{\perp}$. For truly hard diffractive processes, the new model for hard diffraction [9] was developed, not only for high- $p_{\perp}$ jets, but also allowing for $W^{ \pm}, Z^{0}, H$ etc.

The model is based on the assumption that the proton PDF can be split into a diffractive and a nondiffractive part,

$$
f_{i / \mathrm{p}}\left(x, Q^{2}\right)=f_{i / \mathrm{p}}^{\mathrm{ND}}\left(x, Q^{2}\right)+f_{i / \mathrm{p}}^{\mathrm{D}}\left(x, Q^{2}\right),
$$

with the diffractive part being described using the factorisation approach of Ingelman and Schlein,

$$
\begin{aligned}
f_{i / \mathrm{p}}^{\mathrm{D}}\left(x, Q^{2}\right) & =\int_{0}^{1} \mathrm{~d} x_{\mathbb{P}} f_{\mathbb{P} / \mathrm{p}}\left(x_{\mathbb{P}}\right) \int_{0}^{1} \mathrm{~d} x^{\prime} f_{i / \mathbb{P}}\left(x^{\prime}, Q^{2}\right) \delta\left(x-x_{\mathbb{P}} x^{\prime}\right) \\
& =\int_{x}^{1} \frac{\mathrm{d} x_{\mathbb{P}}}{x_{\mathbb{P}}} f_{\mathbb{P} / \mathrm{p}}\left(x_{\mathbb{P}}\right) f_{i / \mathbb{P}}\left(\frac{x}{x_{\mathbb{P}}}, Q^{2}\right),
\end{aligned}
$$

where $f_{\mathbb{P} / \mathrm{p}}\left(x_{\mathbb{P}}\right)=\int_{t_{\min }}^{t_{\max }} \mathrm{d} t f_{\mathbb{P} / \mathrm{p}}\left(x_{\mathbb{P}}, t\right)$, as $t$ for the most part is not needed.

The probability for side $A, B$, to be diffractive is then given by the ratio of diffractive to inclusive PDFs,

$$
\begin{gathered}
\mathcal{P}_{A}^{\mathrm{D}}=\frac{f_{i / B}^{\mathrm{D}}\left(x_{B}, Q^{2}\right)}{f_{i / B}\left(x_{B}, Q^{2}\right)} \text { for } A B \rightarrow X B, \\
\mathcal{P}_{B}^{\mathrm{D}}=\frac{f_{i / A}^{\mathrm{D}}\left(x_{A}, Q^{2}\right)}{f_{i / A}\left(x_{A}, Q^{2}\right)} \text { for } A B \rightarrow A X .
\end{gathered}
$$




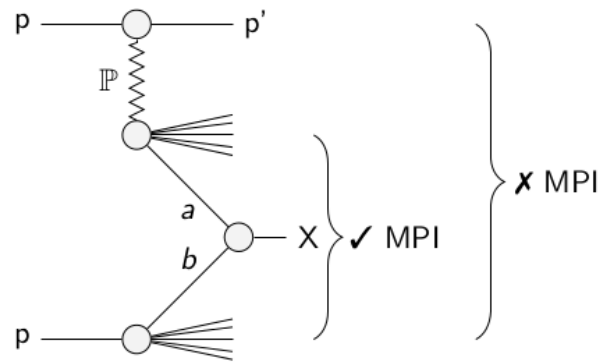

Figure 1. The dynamical gap survival implemented in Pythia 8. The model do not allow for MPIs in the pp system, but allows for additional MPIs in the $\mathbb{P}$ p system.

Table 1. Diffractive fractions for the $2 \rightarrow 2 \mathrm{QCD}$ processes with $p_{\perp}>20 \mathrm{GeV}$ in pp collisions at $\sqrt{s}=8 \mathrm{TeV}$ obtained with Pутнік 8 . The samples have been produced without any phase-space cuts. $\mathbb{P}$ parametrizations not cited in the text are: H1 Fit A NLO PDF [11] and H1 Fit A flux [11, 13]

\begin{tabular}{lll}
\hline $\begin{array}{l}\mathbb{P} \text { PDF } \\
\mathbb{P} \text { flux }\end{array}$ & PDF selection & MPI selection \\
\hline $\begin{array}{l}\text { H1 Fit B LO } \\
\text { Schuler-Sjöstrand }\end{array}$ & $(14.33 \pm 0.11) \%$ & $(0.98 \pm 0.03) \%$ \\
H1 Fit B LO & & \\
MBR & $(14.79 \pm 0.11) \%$ & $(0.96 \pm 0.03) \%$ \\
H1 Jets & & \\
Schuler-Sjöstrand & $(13.70 \pm 0.11) \%$ & $(0.92 \pm 0.03) \%$ \\
H1 Fit A NLO & & \\
H1 Fit A & $(20.55 \pm 0.13) \%$ & $(1.35 \pm 0.04) \%$ \\
H1 Fit B LO & & \\
H1 Fit A & $(18.49 \pm 0.12) \%$ & $(1.32 \pm 0.04) \%$ \\
\hline
\end{tabular}

The model also implements a dynamical gap survival, cf. Fig. 1. On an event-by-event basis we evaluate the possibility for additional MPIs in the pp system, and if no further MPIs are found, then the event is diffractive. Thus initially eq. (3) has no consequence, with all events still handled as being nondiffractive. Only if no additional MPIs are found is the event classified as diffractive, and the $\mathbb{P} p$ system set up. A full evolution is then performed in this subsystem, along with the hadronisation of the colour strings in the event. At this point, the nondiffractive events can be discarded if an exclusively diffractive sample is wanted, otherwise they can be kept for an inclusive sample consisting of both non- and single-diffractive events.

The dynamical gap survival introduces an additions suppression of the diffractive events, such that the total probability for hard diffraction drops from $\sim 10 \%$ to $\sim 1 \%$. In Table 1 we show the number of events passing either the probabilistic criterion of eq. (3) (PDF selection) or both the probabilistic and the dynamical gap survival (MPI selection) for a variety of the $\mathbb{P}$ fluxes and PDFs available in PyтнIA 8. These fractions are very model dependent, of course. They depend both on the $\mathbb{P}$ parametrization and on the free parameters of the MPI framework, although many of the distributions tend to be mainly driven by only one of them. 
Table 2. Diffractive fractions for the $2 \rightarrow 2 \mathrm{QCD}$ processes with $p_{\perp}>20 \mathrm{GeV}$ in $\sqrt{s}=8 \mathrm{TeV}$ pp collisions. Pутнia is run with the Schuler-Sjöstrand flux and the H1 Fit B LO PDF.

\begin{tabular}{lll}
\hline & PDF selection & MPI selection \\
\hline$p_{\perp 0}^{\text {ref }}=1.78$ & $(14.50 \pm 0.11) \%$ & $(0.39 \pm 0.02) \%$ \\
$p_{\perp 0}^{\text {ref }}=2.28$ & $(14.33 \pm 0.11) \%$ & $(0.98 \pm 0.03) \%$ \\
$p_{\perp 0}^{\text {ref }}=2.78$ & $(14.19 \pm 0.11) \%$ & $(2.00 \pm 0.04) \%$ \\
\hline
\end{tabular}

Distributions affected by the modelling of the $\mathbb{P}$ flux and PDF are e.g. the chosen $x_{\mathbb{P}}$ value, hence the mass of the diffractive system, as well as the value of the squared momentum transfer, $t$ and through that the angle at which the proton is scattered. The mass of the diffractive system is shown in Fig. 2a, using the following parametrizations: The Schuler-Sjöstrand flux (SaS) [6], the Minimum Bias Rockefeller lux (MBR) [10], the H1 Fit B LO PDF [11] and the H1 Jets PDF [12]. It is observed that the variation of the $\mathbb{P}$ flux affects the distribution more than the variation of the $\mathbb{P} P D F$.

Those affected by the MPI parameters are several particle distributions such as multiplicities and $p_{\perp}$ spectra. One of these is shown in Fig. $2 \mathrm{~b}$, where the transverse matter profile of the $\mathbb{P}$ is varied. In the end, what really matters is the convolution of the matter profile of the $\mathbb{P}$ and the $p$, resulting in an overlap function, from where the impact parameter $b$, describing the amount of overlap between the $\mathbb{P}$ and $\mathrm{p}$, is chosen. We have implemented three options:

- $b_{\mathbb{P p}}=b_{\mathrm{pp}}$, implying that the $\mathbb{P}$ and $\mathrm{p}$ are equally big and have equal matter profiles.

- $b_{\mathbb{P p}}=\sqrt{b_{\mathrm{pp}}}$, motivated if the $\mathbb{P}$ is a point particle. The variation of the overlap function with $b$ is then that of one proton, not two, giving the square root of the normal variation.

- The final option implemented is picking a completely new $b_{\mathbb{P} p}$, ie. not specifying the size of the $\mathbb{P}$ or its matter profile.

The variation of the size gives different distributions, the latter definition giving much larger multiplicities than the two first (and probably more realistic) cases.

Also the gap survival probability is highly sensitive to the parameters of the MPI model, highlighted in Table 2, where the regulator of the $2 \rightarrow 2$ QCD cross section, $p_{\perp 0}^{\text {ref }}$ has been varied with $\pm 0.5 \mathrm{GeV}$, resulting in a factor of two in the diffractive fractions. This major $p_{\perp 0}^{\text {ref }}$ dependence holds also for many nondiffractive event properties, however; keeping everything else fixed even a variation of $\pm 0.1 \mathrm{GeV}$ would be unacceptable. It would be possible to perform a dedicated diffractive tune, but the same parameters would not be able to describe nondiffractive data. Such a retune is considered.

\section{Conclusions}

We have presented the two frameworks for diffraction present in the general-purpose event generator PYтнIA 8. The soft diffraction framework allows for QCD processes at various $p_{\perp}$ scales, although primarily intended for low- $p_{\perp}$ interactions. It gives a decent description of soft diffraction, although comparisons to data show room for improvement. The new model for hard diffraction allows for nonQCD and very high- $p_{\perp}$ QCD processes in diffractive systems. The effects of the $\mathbb{P}$ parametrizations as well as the free parameters of the MPI framework have been explored. Future work includes a retuning of the free parameters in both models and developing a new parametrization of the $\mathbb{P}$ flux, in order to obtain a better description of data. 


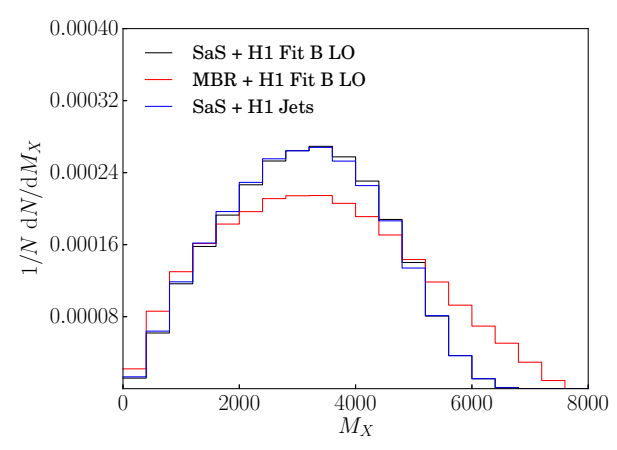

(a)

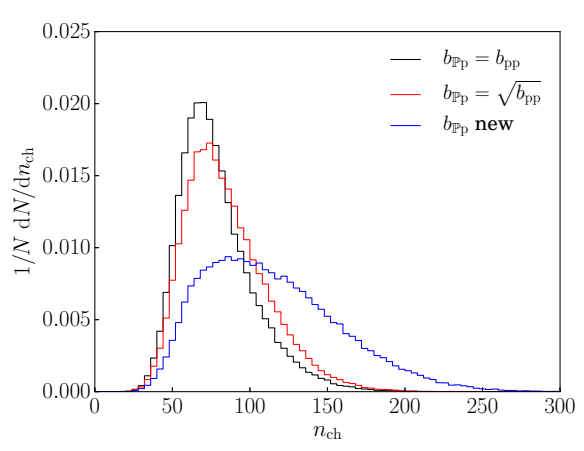

(b)

Figure 2. (a) The mass of the diffractive system, $M_{X}$, when changing $\mathbb{P}$ parametrization. (b) The number of charged particles in the $\mathbb{P}$ system, when changing the size of the $\mathbb{P}$.

\section{Acknowledgements}

Work supported by the MCnetITN FP7 Marie Curie Initial Training Network, contract PITN-GA2012-315877.

\section{References}

[1] T. Sjöstrand et al., Comput. Phys. Commun. 191 (2015) 159 [arXiv:1410.3012 [hep-ph]].

[2] G. Ingelman and P. E. Schlein, Phys. Lett. B 152 (1985) 256.

[3] T. Sjöstrand, S. Mrenna and P. Z. Skands, JHEP 0605 (2006) 026 [hep-ph/0603175].

[4] S. Navin, arXiv:1005.3894 [hep-ph].

[5] A. Donnachie and P. V. Landshoff, Phys. Lett. B 296 (1992) 227 [hep-ph/9209205].

[6] G. A. Schuler and T. Sjöstrand, Phys. Rev. D 49 (1994) 2257.

[7] http://home.thep.lu.se/Pythia/pythia82html/Welcome.html

[8] B. Andersson, G. Gustafson, G. Ingelman and T. Sjöstrand, Phys. Rept. 97 (1983) 31.

[9] C. O. Rasmussen and T. Sjöstrand, in preparation.

[10] R. Ciesielski and K. Goulianos, PoS ICHEP 2012 (2013) 301 [arXiv:1205.1446 [hep-ph]].

[11] A. Aktas et al. [H1 Collaboration], Eur. Phys. J. C 48 (2006) 715 [hep-ex/0606004].

[12] A. Aktas et al. [H1 Collaboration], JHEP 0710 (2007) 042 [arXiv:0708.3217 [hep-ex]].

[13] A. Aktas et al. [H1 Collaboration], Eur. Phys. J. C 48 (2006) 749 [hep-ex/0606003]. 